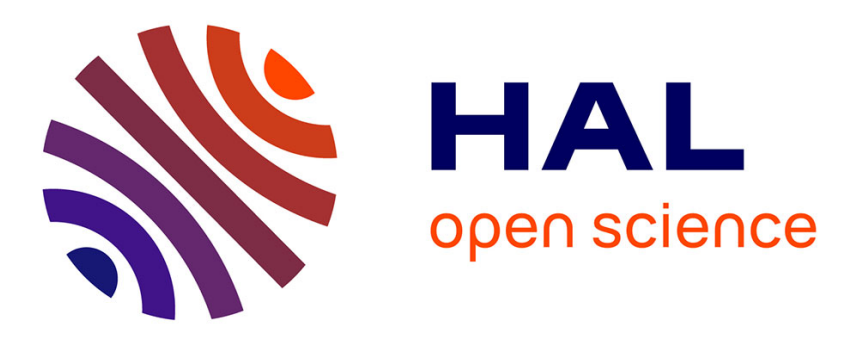

\title{
Naissance de l'économie politique, idéologie propriétariste et Révolution française
}

\author{
Arnaud Orain
}

\section{To cite this version:}

Arnaud Orain. Naissance de l'économie politique, idéologie propriétariste et Révolution française. 2021. hal-03376758

\author{
HAL Id: hal-03376758 \\ https://hal.science/hal-03376758
}

Preprint submitted on 13 Oct 2021

HAL is a multi-disciplinary open access archive for the deposit and dissemination of scientific research documents, whether they are published or not. The documents may come from teaching and research institutions in France or abroad, or from public or private research centers.
L'archive ouverte pluridisciplinaire HAL, est destinée au dépôt et à la diffusion de documents scientifiques de niveau recherche, publiés ou non, émanant des établissements d'enseignement et de recherche français ou étrangers, des laboratoires publics ou privés. 


\title{
Naissance de l'économie politique, idéologie propriétariste et Révolution
}

\section{française}

\author{
Arnaud ORAIN \\ Naissance de l'économie politique, idéologie propriétariste et Révolution \\ française
}

Cet article discute et prolonge les éléments d'histoire intellectuelle avancés par Thomas Piketty dans la première partie de Capital et idéologie autour du passage d'une société trifonctionnelle (noblesse, clergé, tiers état) à une société propriétariste dans la France du XVIII ${ }^{\mathrm{e}}$ siècle. Il s'intéresse en particulier à la dichotomie entre deux conceptions de l'économie politique à sa naissance (transcendance contre contingence), qui renvoie au partage entre partisans et adversaires de la naturalisation de la propriété, du marché, des inégalités et de la science. L'article tente également d'expliquer la victoire de la logique propriétariste, qui est en même temps celle de la physiocratie, sous la Révolution française.

MOTS-CLES : Ordre naturel, Transcendantaux, Révolution française, Biens nationaux

Abstract

Arnaud ORAIN

The Birth of Political Economy, Proprietary Ideology and the French Revolution

This article discusses and extends several elements of intellectual history put forward by Thomas Piketty in the first part of Capital and Ideology related to the shift from a trifunctional society (nobility, clergy, third estate) to a proprietary society in $18^{\text {th }}$ century France. The paper highlights the dichotomy between two conflicting approaches of political economy at its birth (transcendence Vs contingence), which refers to the great debate among supporters and opponents of the naturalization of ownership, markets, inequalities and science. It is also an attempt to explain the success of the proprietary logic, which is at the same time that of Physiocracy, during the French Revolution.

KEYWORDS: Natural Order, Transcendentals, French Revolution, Biens nationaux 
Le $1^{\text {er }}$ février 1793, à la tribune de la Convention, le député Cambon s'exprime au nom du comité des Finances sur la situation budgétaire. Tandis qu'aucun impôt ne rentre dans les caisses de l'État, les dépenses sont assurées par la création d'assignats gagés sur les domaines confisqués à l'Église. Cambon en tire une interprétation eschatologique de l'œuvre révolutionnaire :

«Cette recette est le produit de l'aliénation des capitaux qui étaient entre les mains de cette caste privilégiée qui, portant le Christ d'or à la main, arrachait le produit de la sueur des peuples. Ils étaient réservés pour opérer la plus belle révolution du monde ; nous les avons employés à assurer la liberté et l'égalité, et à nous rendre tous frères et égaux. C'était la doctrine que les prêtres voluptueux prêchaient ; leurs vœux doivent être remplis puisque leurs biens ont servi à réaliser ce qu'ils annonçaient depuis tant de siècles ${ }^{1}$.

Si l'on en croit Cambon, la Révolution était en train d'opérer une profonde redistribution des richesses, à la fois par le démembrement des grandes propriétés foncières et par un désendettement massif rendu possible par les remboursements en assignats dépréciés. Il ne manquait plus qu'une pression fiscale très faible pendant près d'une décennie (17891799), et le Code civil de 1804 avec son principe de partage égalitaire des successions, pour que s'installe, au XIX ${ }^{\mathrm{e}}$ siècle, le mythe d'un pays de petits propriétaires fonciers, devenu faiblement inégalitaire à la suite des événements révolutionnaires. Si les lecteurs de Balzac, et certains historiens, se doutaient que les choses étaient un peu plus compliquées, il revient à Thomas Piketty d'avoir mis en exergue l'ampleur du décalage entre le mythe et la réalité.

L'un des premiers résultats importants de Capital et idéologie concerne en effet la concentration des revenus et de la propriété. La comparaison est saisissante : à la veille de la Première Guerre mondiale, les inégalités sont équivalentes - voire supérieures - à ce qu'elles étaient dans les années 1780. Les $10 \%$ les plus riches possèdent toujours entre 80 et $90 \%$ des propriétés privées et perçoivent plus de $50 \%$ du total des revenus (p. 163). Mais Thomas Piketty ne s'en tient pas à ce constat chiffré. Il s'attache à la construction intellectuelle qui a rendu possible un tel état de fait, avec un deuxième résultat, plus remarquable encore que le premier : l'attachement indéfectible à la propriété privée des révolutionnaires de 1789 - et on pourrait ajouter, la plupart de ceux de 1793 - a non seulement empêché une véritable redistribution des richesses à la fin du XVIII ${ }^{\mathrm{e}}$ siècle, mais il a encore préparé l'avènement de la société violemment inégalitaire du siècle suivant.

\footnotetext{
${ }^{1}$. Rapport et projet de décret présentés à la Convention nationale, au nom du comité des finances, par le Citoyen Cambon, Paris, Imprimerie Nationale, 1793, p. 8.
} 
L'un des aspects les plus intéressants de la première partie du livre concerne en effet les modalités du passage d'une société d'ordres trifonctionnelle (clergé, noblesse, tiers-état), à une autre fondée sur la logique propriétariste. Ma discussion du travail de Thomas Piketty porte principalement sur ce point, l'idée étant d'approfondir le lien esquissé dans l'ouvrage (p. 147-148; p. 745-746) entre la «naturalisation» des inégalités et l'émergence concomitante d'une économie politique aux fondements théoriques «naturels ». En opposant le philo-physiocrate Condorcet d'un côté, très timide en matière fiscale, et l'antiphysiocrate Graslin de l'autre, qui propose une forte progressivité de l'impôt, Piketty nous invite à revenir sur le débat décisif qui s'est produit en France autour de la «science nouvelle », lors des décennies 1760-1770². Mon propos est ici de tirer les fils d'histoire intellectuelle que Thomas Piketty suggère, pour faire apparaître deux éléments. Le premier concerne l'opposition entre une conception transcendante et une autre, contingente, de l'économie politique à sa naissance, au XVIII ${ }^{\mathrm{e}}$ siècle. Cette dichotomie renvoie au partage entre partisans et adversaires de la naturalisation de la propriété, du marché et de la science, dont découlent des propositions divergentes en matière de régulation des inégalités. Le second élément sur lequel je souhaite insister concerne la victoire de la logique propriétariste, qui est en même temps celle de la physiocratie. Alors que celle-ci semblait avoir été vaincue dans l'Ancien Régime, nombre de ses idées politiques triomphent lors de la Révolution française. Il reste à tenter de comprendre comment, et pourquoi il en a été ainsi.

\section{LE PARTAGE DES EAUX (ECONOMIQUES) : TRANSCENDANCE CONTRE CONTINGENCE}

Thomas Piketty souligne à la fois l'analogie et la tension entre la sacralisation de la propriété privée, d'une part, et l'utopie d'une réduction des inégalités par la libéralisation des marchés d'autre part. Les deux idées reposent sur des conceptions transcendantes ou « naturelles » des fondements de la société. D'un côté, en effet, «le respect absolu des droits de propriété acquis dans le passé fournit une transcendance nouvelle permettant [...] de remplir le vide laissé par la fin de l'idéologie trifonctionnelle. La sacralisation de la propriété est d'une certaine façon une réponse à la fin de la religion comme idéologie politique explicite» (p. 155). De l'autre, l'optimisme des partisans des «forces naturelles », les conduisait à penser qu'il suffirait «de supprimer les privilèges et les charges, d'établir

\footnotetext{
2. Steven Kaplan, Sophus Reinert (éd.), The Economic Turn: Recasting Political Economy in EighteenthCentury Europe, London, Anthem Press, 2019 ; Gérard KLOTZ, Arnaud ORAIN et Philippe MinARD (éd.), Les voies de la richesse ? La physiocratie en question (1760-1850), Rennes, Presses Universitaires de Rennes, 2017.
} 
l'égalité d'accès aux différents métiers et au droit de propriété pour que les inégalités anciennes disparaissent » (p. 148). Capital et idéologie montre de manière éclatante qu'il n'en est rien, car le propriétarisme a longtemps empêché toute forme de redistribution. Mais on peut aller plus loin et revenir sur le grand partage qui a opposé, en France, les partisans de cette nouvelle transcendance aux défenseurs d'une conception contingente et historique de l'économie politique et de la société. Cette généalogie est d'autant plus importante à établir que ce débat, au cœur des Lumières, renvoie à la façon dont Thomas Piketty nous invite à repenser aujourd'hui les savoirs économiques.

Née à la fin des années 1750 dans un contexte de bouillonnement intellectuel, la théorie physiocrate repose sur une transcendance, celle de l'« ordre naturel». Ainsi pour ses pères fondateurs, Quesnay et Mirabeau,

«le gouvernement parfait n'est pas d'institution humaine (...), «les hommes ne peuvent rien ajouter ni rien retrancher à cette THEOCRATIE, leur bonheur consiste à s'y conformer. Le Gouvernement des Princes n'est pas, comme on le croit vulgairement, l'art de conduire les hommes ; c'est l'art de pourvoir à leur sureté et à leur subsistance par l'observation de l'ordre naturel $»^{3}$.

Ce dernier, pour leur disciple Lemercier de la Rivière, est une nécessité : «Le Législateur universel n'a point laissé aux hommes le soin d'instituer des lois à cet égard ; ce même ordre est au contraire tout naturellement établi tel qu'il doit être dans toutes les sociétés du monde entier ; aussi se maintiendra-t-il toujours et nécessairement. » À partir de cet ordre, les physiocrates naturalisent deux éléments : la propriété privée et les forces du marché : «Propriété, et par conséquent sûreté et liberté de jouir, voilà donc ce qui constitue l'essence de l'ordre naturel », affirme Lemercier ${ }^{4}$.

Lors des terribles événements qui secouent le royaume à la suite de la libéralisation du commerce des grains en 1768-1770, puis de nouveau en 1775 - disettes, émeutes, répressions - les théoriciens physiocrates campent sur une ligne dure. Contre ceux qui souhaiteraient voir le gouvernement réquisitionner les grains et contrôler les prix, ils expliquent qu' « on ne pourrait, sans tomber dans une contradiction choquante, s'armer du prétexte de l'intérêt commun, pour porter atteinte au droit de propriété foncière qui en est la base ». Selon eux, « tous les droits résultent du droit de la propriété », car comme l'a défendu Quesnay en 1765, «la sûreté de la propriété privée » est «le fondement essentiel de l'ordre économique de la

\footnotetext{
${ }^{3}$. François QUESNAY et Victor RIQUETI DE MIRABEAU, Philosophie rurale, Amsterdam, Les Libraires Associés, 1763 , t. I, p. xlij.

4. Paul-Pierre LEMERCIER DE LA RIVIERE, L'ordre naturel et essentiel des sociétés politiques, Londres et Paris, Nourse et Desaint, 1767, t.I, p. 271 et 59.
} 
société ». Il s'ensuit que les inégalités ne peuvent être remises en cause par l'État, car le risque est trop grand de spolier des propriétaires légitimes. Ni bonnes ni mauvaises en ellesmêmes, ces inégalités résultent «de la combinaison des lois de la nature ; et les hommes ne pouvant pénétrer les desseins de l'être suprême dans la construction de l'univers, ne peuvent s'élever jusqu'à la destination des règles immuables qu'il a instituées pour la formation et la conservation de son ouvrage $»^{5}$.

Mais ce n'est pas tout : l'« ordre naturel » contient encore un principe d'organisation du monde qui échappe au pouvoir des êtres humains. Ainsi Lemercier explique-t-il que

«l'intérêt personnel [...] presse vivement et perpétuellement chaque homme en particulier, [...] de multiplier les choses dont il est vendeur, de grossir ainsi la masse des jouissances qu'il peut procurer aux autres hommes, afin de grossir, par ce moyen, la masse des jouissances que les autres hommes peuvent lui procurer en échange. Le monde, alors, va de lui-même [dans] un mouvement qui devient une tendance perpétuelle vers son meilleur état possible ${ }^{6}$.

L'ordre est une entité autorégulatrice qui institue les sociétés, puis les porte vers la prospérité matérielle, alors que chacun ne cherche qu'à poursuivre son intérêt personnel. Comme le précise Morellet, compagnon de route de la physiocratie, « de l'opposition même des intérêts particuliers, naît le bien public ; et que jusqu'aux vices de chaque homme, [...], tout concourt à l'harmonie de la société $»^{7}$. Ces théoriciens pensent que lorsque les principes de l'ordre naturel sont respectés - propriété privée inviolable et liberté totale du commerce (suppression des corporations, des douanes, des péages, etc.) -, les inégalités devraient avoir tendance à diminuer.

Si Lemercier ne cesse de répéter que cet « ordre » est accessible à la raison humaine, ce n'est pourtant pas le cas. Jamais il n'explique pourquoi, si on laisse faire la « nature », il en résulterait une harmonie sociale. Et pour cause : ce point est inexplicable, à moins de croire que «l'ordre » existe et que grâce à lui «le monde va de lui-même ». C'est donc soit une tautologie, soit un postulat, ce que Lemercier est forcé de reconnaître par endroits : «1'ordre naturel immuable » est «institué par l'Auteur de la nature pour gouverner les hommes $»^{8}$. La science des physiocrates et ses deux « lois » (propriété sacrée et marché autorégulé) sont par

\footnotetext{
5. P.-P. LEMERCIER DE LA RIVIERE, L'intérêt général de l'État, Amsterdam et Paris, Desaint, 1770, p. 47 ; PierreJoseph RoubAud, Récréations économiques, Amsterdam et Paris, Delalain et Lacombe, 1770, p. 29 ; F. QUESNAY, « Maximes générales du gouvernement économique d'un royaume agricole » (1767-8), in QUESNAY, Euvres économiques complètes et autres textes, Paris, INED, 2005, t. I, p. 567 ; «Droit naturel » (1765), in Ibid, p. 115.

6. P.-P. LEMERCIER DE LA RIVIERE, L'ordre naturel, op. cit., II, p. 447.

7. André MoRellet, Réfutation de l'ouvrage qui a pour titre Dialogues sur le commerce des bleds, Londres, 1770, p. 110.

${ }^{8}$ P.-P. LEMERCIER DE LA RIVIERE, L'ordre naturel, op. cit., II, p. 424.
} 
conséquent fondées sur une vérité métaphysique et transcendantale. C'est précisément ce que combattent au même moment plusieurs philosophes et économistes majeurs des Lumières européennes.

Le diplomate et économiste napolitain Ferdinando Galiani, adversaire résolu des physiocrates, explique ainsi en 1770 que l'idée selon laquelle «la nature en liberté » tendrait «à l'équilibre », « est une vérité lumineuse dans la tête d'un métaphysicien », mais que cela est «très faux sous la main d'un praticien ». Son ami Diderot répond à Morellet que « rien ne va seul dans aucun pays au monde $»^{9}$. Mais dans ce combat antiphysiocrate des années 1760 , un homme a joué un rôle décisif, l'économiste François Véron de Forbonnais. Ancien membre éminent du cercle de Gournay dans les années 1750, négociant et propriétaire foncier devenu homme de lettres et conseiller de plusieurs ministres, Forbonnais récuse l'idée selon laquelle il faudrait fonder une «science nouvelle»sur des bases métaphysiques. Selon lui, il existe déjà un savoir économique suffisant développé à partir de cas pratiques et de «vérités locales », et il n'aura de cesse de dénoncer les prétendues découvertes de Quesnay et de ses disciples comme des mystifications et des impostures ${ }^{10}$.

Sans remettre en cause son existence, Forbonnais, pense ainsi qu'il est impossible de fonder quoi que ce soit sur un ordre naturel : «Quelques philosophes », dit-il, ont voulu « ramener la pratique économique uniquement aux règles qui découlent de l'ordre naturel. » Or,

« cet ordre n'a jamais pu ni dû être altéré dans son essence; mais ses conséquences ont été modifiées par des circonstances qui intéressent la conservation des sociétés. C'est donc sous ce double point de vue que les principes doivent être envisagés, calculés, et suivis [...]. Il ne serait donc pas plus raisonnable d'administrer la partie économique d'un État, [...] par les principes de l'ordre naturel des choses, que de sacrifier les hiérarchies établies dans la constitution, à l'idée purement philosophique de l'égalité des conditions $\gg^{11}$.

L'histoire, les relations internationales, le droit public et privé, les constitutions ont modifié l'ordre naturel. Ce point est crucial : pour Galiani, Diderot, Forbonnais et quelques autres, il existe peut-être, en abstraction, un « ordre » qui règle les rapports entre les choses et les individus, mais il est impensable de construire une science à partir de lui. Ces auteurs refusent la métaphysique physiocrate et ses prétendues lois naturelles, pour au contraire

\footnotetext{
${ }^{9}$ Ferdinando Galiani, Dialogues sur le commerce des bleds, Londres, 1770, p. 236-237; Denis DiDERot, «Apologie de l'abbé Galiani », in DiDEROT, Euvres, Paris, Robert Laffont, 1995, t. III, p. 146.

${ }^{10}$ Loïc CHARLES et Arnaud ORAIN, « François Véron de Forbonnais and the Invention of Antiphysiocracy », in KAPLAN and REINERT (éd.), The Economic Turn, op. cit., p. 139-168.

${ }^{11}$ François VERON DE ForbonNAIS, Principes et observations économiques, Amsterdam, Marc-Michel Rey, 1767, t.I, p. 147-148.
} 
penser le monde dans sa contingence. De ce point de vue, la citation de Forbonnais autour de l'objet qui nous occupe ne doit pas tromper : s'il s'oppose à la stricte égalité des conditions, il est parfaitement en accord avec Galiani pour ne pas sacraliser la propriété privée ${ }^{12}$. La puissance publique doit non seulement intervenir pour réguler le marché en temps de disette, mais elle doit aussi le faire en temps normal. En s'intéressant aux réalités du commerce des céréales (pouvoirs locaux, voies de communication, comportements des acteurs, etc.), Galiani et Forbonnais envisagent une liberté du commerce encadrée (prix-plafond à l'exportation, transport obligatoire sur des navires français, droits de douane). Ils ne croient pas à l'idée selon laquelle «le monde ira de lui-même » si on laisse faire les forces concurrentielles.

C'est Kant qui a posé le jugement le plus perspicace sur la doctrine de Quesnay et de ses disciples, lorsqu'il a parlé de "physiocratie transcendantale». En affirmant «la toute puissance de la nature », ces théoriciens ont placé hors de l'expérience sensible un pouvoir qui ne peut pas être interrogé par l'entendement humain, celui de l'ordre naturel. Or comme l'explique Foucault, c'est précisément par l'établissement de transcendantaux, extérieurs mais fondateurs, que les sciences modernes - économie politique comprise - sont nées à la fin du $\mathrm{XVIII}^{\mathrm{e}}$ siècle $^{13}$. Il y a là un point majeur qui entre en écho avec les critiques que Thomas Piketty adresse à la science économique. Dès sa fondation au cœur des Lumières, des théoriciens ont farouchement refusé qu'elle repose sur une base transcendantale. Ils ont construit un savoir économique contingent, en s'intéressant à l'histoire du commerce, à la géographie physique, aux lois, aux traités, et aux observations. Ils n’ont envisagé les questions économiques que d'un point de vue déterministe - selon les «climats » et les particularités régionales, autrement dit les données empiriques et historiques d'un lieu situé. Très méfiant vis-à-vis du pouvoir heuristique des abstractions, insistant selon le terme de Forbonnais sur les seules «vérités locales », ces économistes ont souvent mis en doute les possibilités même de principes et de politiques économiques réplicables. Depuis lors, les commentateurs ont eu le plus grand mal à les situer, et ils ont été engloutis dans une gangue pré-scientifique tombée dans l'oubli. Or si les physiocrates sont à l'origine de la naturalisation

\footnotetext{
${ }^{12}$ Même s'ils combattent à la fois la libéralisation du commerce des grains et la naturalisation des inégalités, Rousseau et Mably reconnaissent l'importance de la propriété privée. Le premier déclare qu' « [i]l est certain que le droit de propriété est le plus sacré de tous les droits des citoyens » (Jean-Jacques RoUSSEAU, Discours sur l'Économie Politique, in Euvres Complètes, Paris, Gallimard, 1954, t.III, p. 262) et le second que « [d]ès que la communauté des biens ne subsiste plus [...], je sais qu'il n'y a point de loi plus sacrée que celle de la propriété » (Gabriel Bonnot DE MABly, Du Commerce des Grains, in Collection complète des CEuvres de l'Abbé de Mably, Paris, Desbrière, 1794-5 [1775], vol. 13, p. 272). Voir KAPLAN and REINERT (éd.), The Economic Turn, op. cit.

${ }^{13}$ Emmanuel KANT, Critique de la raison pure, Paris, Aubier, 1998 [1787], p. * ; Michel FouCAULT, Les mots et les choses, Paris, Gallimard, 1966, p. 256-257.
} 
de la science économique jusqu'à nos jours ${ }^{14}$, ses opposants sont à l'inverse les précurseurs de ce savoir économique immergée dans les sciences sociales que Thomas Piketty appelle de ses vœux (p. 1196-1198).

Pour revenir à la question des inégalités, il faut s'intéresser aux implications des théories fiscales de Forbonnais et de Graslin, à peu près identiques. Thomas Piketty a tiré d'un injuste oubli la théorie de l'impôt progressif du second (p. 140-142), plus développée que toutes celles de ses contemporains. Or les idées fiscales de Graslin et celles de Forbonnais sont au service d'une volonté de remodeler la société dans une optique qui, à deux siècles et demi d'écart, n'est pas sans écho avec celle de l'auteur de Capital et idéologie. Les deux antiphysiocrates imaginent en effet que l'impôt progressif conduira à modifier l'allocation des ressources dans un sens qu'ils jugent positif. En provoquant la diminution de la consommation de luxe, cette fiscalité conduira le capital et le travail vers la terre et les manufactures utiles au plus grand nombre. Les prix des objets de consommation courante devraient baisser et l'emploi augmenter dans ces secteurs, au bénéfice des travailleurs pauvres. Graslin et Forbonnais ont un grand mépris pour les riches propriétaires et les rentiers oisifs. Leur «ivresse », dit Forbonnais, « ainsi que l'enfance, aime le dégât et le ravage » et Graslin de renchérir en disant qu' " aussi étrangers à leur sol qu'à leur patrie, ils portent dans les villes le fardeau de leur oisiveté, et ne sont qu'une charge pour la Nation $»^{15}$. Le législateur doit réprimer leurs comportements destructeurs, subordonner leurs «besoins factices » et leur liberté à l'intérêt du plus grand nombre ${ }^{16}$. Comment ne pas faire un parallèle avec les propos de Thomas Piketty sur la transition écologique ? Celui-ci explique en effet qu'on «ne pourra jamais aller vers la sobriété énergétique si l'on n'a pas une forme de sobriété sociale et économique »: «[t]ant qu'une minorité pourra prendre son jet privé et émettre des quantités de carbone considérables », les plus modestes n'accepterons pas de

\footnotetext{
${ }^{14}$ Michel Foucault, Sécurité, Territoire, Population, Paris, Gallimard- Seuil-EHESS, 2004 et Naissance de la biopolitique, Paris, Gallimard-Seuil-EHESS, 2004 ; Bernard HARCOURT, The Illusion of Free Markets. Punishment and the Myth of Natural Order, Cambridge (Mass.), Harvard University Press, 2011.

${ }^{15}$ F. FORBOnNAIS, Principes, op. cit., p. 41 ; Jean-Joseph-Louis GRASLIN, Essai analytique sur la richesse et sur l'impôt, Londres, 1767, p. 156. Sur les tentatives de mise en œuvre d'impôts progressifs par Forbonnais, voir Arnaud ORAIN, «Soutenir la guerre et réformer la fiscalité. Silhouette et Forbonnais au Contrôle général des finances (1759) », French Historical Studies, 36-3, 2013, p. 417-447.

${ }^{16}$ Arnaud ORAIN, «Progressive indirect taxation and Social Justice in Eighteenth Century France. Forbonnais and Graslin's Fiscal System. » The European Journal of the History of Economic Thought, 17-4, 2010, p. 657683. Sur l'idée de régénération morale par l'impôt au siècle des Lumières, voir Nicolas DELALANDE, " Gouverner les conduites par la fiscalité ? Une brève histoire des débats sur le pouvoir incitatif de l'impôt ", in Sophie Dubuisson-Quellier (éd.), Gouverner les conduites, Paris, Presses de Sciences Po, 2016, p. 59-92.
} 
changer de comportement ${ }^{17}$. Il s'agit bien de subordonner la liberté et le droit de propriété d'une minorité à la prospérité de la majorité.

\section{LA REVOLUTION FRANÇAISE OU LE TRIOMPHE POSTHUME DE LA PHYSIOCRATIE}

Le constat de Thomas Piketty semble sans appel : la Révolution française n'a pratiquement pas réduit les inégalités économiques. Bien sûr une partie des patrimoines a changé de mains. Le Clergé a presque tout perdu et la part des propriétés foncières détenue par les nobles a reflué dans les années 1790-1800. Mais ces derniers sont non seulement loin d'avoir été ruinés par la Révolution, leurs patrimoines, notamment immobiliers, se sont fortement accrus au cours des décennies 1810-1840 (p. 112-116). Ainsi la concentration de la propriété privée est-elle à peine plus faible en 1810 qu'en 1780, puis n'a de cesse de progresser ensuite (p. 160-163). Si les cartes des richesses ont été un peu redistribuées, c'est entre personnes aisées, voire opulentes, que le jeu s'est principalement déroulé.

L'ampleur des transferts de propriété foncière au cours de la Révolution doit d'abord être relativisée : sans doute moins de $10 \%$ de la totalité des terres. On doit relever ensuite que les bourgeois des villes l'emportent très nettement dans les achats de biens nationaux sur les ruraux, et que chez ces derniers les gros fermiers étaient majoritaires lors des acquisitions. Enfin il faut souligner que l'abolition des droits féodaux et la clarification de la notion de propriété ont accéléré le marché de la terre, au grand bénéfice des plus riches. La Constituante et la Législative ont favorisé ces derniers car les biens confisqués à l'Église étaient vendus aux enchères et dans leur entièreté (par exemple une abbaye avec son domaine foncier). Si quelquefois les paysans se coalisèrent pour acheter et bloquèrent les adjudications, il s'agissait de faits sporadiques et marginaux. La Convention autorisa le découpage de ces biens en lots plus petits et elle tenta de légiférer pour que les indigents puissent en acquérir. L'application de ces mesures fut cependant variable sur le territoire national, et de courte durée. Contre les radicaux des sections parisiennes, ils votèrent le 18 mars 1793 à une très large majorité un décret qui punissait de mort tous ceux qui proposeraient une loi agraire ou toute autre loi subversive des propriétés. Quelles qu'aient été leurs divisions par ailleurs, Girondins et Montagnards étaient fondamentalement d'accord sur le fait que la propriété privée devait constituer la base de la société. Ainsi les seconds ont-ils encouragé, par exemple, le partage des communaux dans le but de favoriser l'accès à la terre des paysans

\footnotetext{
${ }^{17}$ Thomas PIKETTY, interview au journal La Tribune, 15 novembre 2019.
} 
pauvres. Cette entreprise a toutefois été engagée dans le strict cadre de l'idéologie propriétariste, et parfois au détriment de ceux qui vivaient jusque-là dans les interstices de la société et de ses lois. Le Code Rural de la Constituante et de la Législative, qui donnent les pleins pouvoirs aux propriétaires sur leurs terres, n'a pas été modifié par la Convention jacobine, et ses dispositions sont sorties renforcées du Directoire. Hormis chez les radicaux de l'égalité des conditions - qui ne siégeaient pas dans les différentes assemblées - une véritable réforme agraire n'a jamais été à l'ordre du jour ${ }^{18}$.

L'abolition des privilèges lors de la nuit du 4 août 1789, rappelle Thomas Piketty en s'appuyant sur l'ouvrage de Rafe Blaufarb, amorce le grand partage entre pouvoir régalien et droit de propriété (p. 128-130) ${ }^{19}$. Cette entreprise titanesque devait donner lieu à de multiples discussions que l'auteur de Capital et idéologie retrace avec acuité, notamment dans le cas des «lods » (p. 134-138), mais on peut s'intéresser plus avant à sa généalogie intellectuelle. On ne s'étonnera pas de voir le mouvement physiocrate en pointe sur cette question.

Dans ses Lettres sur la législation (1775), Mirabeau rappelle que la propriété privée est selon la doxa de l'école « la loi fondamentale de la société ». Il souhaite cependant aller plus loin. Il faut regarder, dit-il, les surcharges que subit la terre « de cens, de droits et de redevances, qui sont le titre primordial de la tradition ancienne des terres en Europe, comme une preuve de barbarie de ces âges d'inféodation », devenues au XVIII ${ }^{\mathrm{e}}$ siècle un «brigandage étudié et déguisé, cent fois plus désastreux encore ». Un an plus tard, un commis de Turgot, alors Contrôleur général des finances, fait paraître avec la bienveillante approbation du ministre un ouvrage intitulé Inconvénients des droits féodaux. Dans cet opuscule, Boncerf explique que «le vœu de la société, qui est la meilleure culture possible » exige «la propriété la plus parfaite, la tranquillité la plus profonde, que les droits féodaux détruisent ou empêchent absolument». Le livre est condamné à être brûlé et le Parlement de Paris en profite pour dénoncer le parti physiocrate comme voulant subvertir la société. Cela n'empêche pas un autre disciple, Le Trosne, de demander au même moment que les droits

\footnotetext{
${ }^{18}$ Georges LEFEBVRE, «La place de la Révolution dans l'histoire agraire de la France », Annales HES, 1-4, 1929, p. 506-523 ; Gérard BEAUR, « Révolution et redistribution des richesses dans les campagnes : mythe ou réalité ? », AHRF, 352, 2008, p. 209-239 ; Bernard BODINIER, "La Révolution française et la question agraire », Histoire \& Sociétés rurales, 33-1, 2010, p. 7-47; Laurent BRASSART et alii, «Terre et agriculture sous la Révolution et l'Empire », AHRF, 382, 2015, p. 145-155 ; Jean-Pierre JESSENNE et Nadine VIVIER, «Libérer la terre ! Une Europe des réformes agraires (vers 1750-1850) ? », RHMC, 63-4bis, 2016, p. 27-65.

${ }^{19}$ Rafe BlaUfarb, L'invention de la propriété privée. Une autre histoire de la Révolution, Ceyzérieu, Champ Vallon, 2019 [2016].
} 
féodaux soient abolis pour rendre «la propriété pleine, entière et absolument exclusive $»^{20}$. Les exemplaires de son livre, imprimé clandestinement à Bâle, sont saisis par le garde des sceaux qui craint des remous.

Il est vrai que les physiocrates se refusent depuis longtemps déjà à raisonner en termes d'ordres ou de privilèges, et plus généralement à partir d'une conception organiciste de la monarchie. Sous leurs plumes, la société trifonctionnelle a disparu pour laisser place à un nouveau découpage entre propriétaires et non-propriétaires. La distinction fonde l'ordre politique, puisque les premiers sont invités à exercer le pouvoir dans les institutions décentralisées que les physiocrates appellent de leurs vœux, assemblées provinciales et municipalités $^{21}$. Seuls en mesure de voter et d'être élus, les propriétaires fonciers sont également les seuls à être soumis à la fiscalité à travers le fameux «impôt unique » sur le produit net des terres, qui ne doit pas épargner les privilégiés. Les physiocrates veulent en effet balayer le système fiscal de la monarchie : ils se prononcent clairement contre les impôts indirects, contre ceux assis sur le revenu, et ne proposent pas de progressivité ${ }^{22}$.

Thomas Piketty rappelle (p. 152-153) que la «propriété » est transformée en droit naturel dans l'article 2 de la Déclaration des droits de l'homme et du citoyen de 1789 : «Le but de toute association politique est la conservation des droits naturels et imprescriptibles de l'Homme. Ces droits sont la liberté, la propriété, la sûreté, et la résistance à l'oppression. » Quant à l'article 17, le dernier, il renforce son caractère transcendant en qualifiant la propriété de «droit inviolable et sacré ». On sait que parmi les groupes d'influence qui participèrent à la rédaction de la Déclaration, la Société des Trente fut l'un des principaux. Or on trouve en son sein le physiocrate Dupont et les amis du mouvement que sont Condorcet, le duc de la Rochefoucault d'Enville (intime de Turgot), ainsi que le comte de Mirabeau (fils du marquis). Ce dernier n'hésite d'ailleurs pas, lors du débat sur l'article 2, à expliquer à la barre de l'Assemblée que «ce paragraphe est une déclaration de droit tout entière. Tout est dans ce principe si élevé, si libéral, si fécond, que mon père et son illustre ami, M. Quesnay, ont

\footnotetext{
${ }^{20}$ V. MIRABEAU, Lettres sur la législation, Berne, Société typographique, 1775, t. II, p. 413, 444-445; PierreFrançois BONCERF, Inconvénients des droits féodaux, Paris, 1776, p. 66; Guillaume-François LE TROSNE, De l'administration provinciale et de la réforme de l'impôt, Basle, 1779, p. 634.

${ }^{21}$ Le Trosne explique qu'une «administration de propriétaires [...] est donc la forme la plus honorable pour le Souverain et pour la Nation» (G.-F. LE TROSNE, De l'administration provinciale, op. cit., p. 318). Voir Keith BAKER, Inventing the French Revolution. Essays on French Political Culture in the Eighteenth Century, Cambridge, CUP, 1990, p. 239-242; Anthony MERGEY, L'État des physiocrates : autorité et décentralisation, Aix-en-Provence, PUAM, 2007, p. 216-221; Loïc ChARLES et Philippe STEINER, «Entre Montesquieu et Rousseau. La Physiocratie parmi les origines intellectuelles de la Révolution française », Études Jean-Jacques Rousseau, 11, 1999, p. 124-125.

${ }^{22}$ Des expériences - modestes - d'impôts progressifs sur le revenu sont tentées à la fin de l'Ancien régime. Voir Mireille TOUZERY, «Les origines de l'impôt sur le revenu en France : de la monarchie aux républicains radicaux (XVIII ${ }^{\mathrm{e}}$-XIX ${ }^{\mathrm{e}}$ siècles), Revue belge de philologie et d'histoire, 75-4, 1997, p. 1027-1044.
} 
consacré il y a trente ans [...], tous les devoirs de l'homme en dérivent $»^{23}$. Les choses sont dites clairement et il est exact que cet article reprend presque mot pour mot les phrases de Lemercier de la Rivière que j'ai cité précédemment.

Le débat autour de la création des assignats permet de voir combien cette rhétorique physiocratisante a infusé dans les rangs des constituants ${ }^{24}$. Dupont s'y oppose au nom du droit de propriété, ainsi que d'autres membres des Trente, comme le lyonnais Bergasse. Prévoyant que les créanciers seront remboursés en assignats dépréciés, ce qui reviendra à les flouer, Bergasse explique que dans son mandat il lui était «enjoint expressément de ne pas souffrir qu'il fut porté atteinte aux droits de propriété ». Son compatriote du Forez, le député Delandine, explique pour sa part que « ces papiers viendront refluer sur les véritables familles de l'État, sur les propriétaires ». «On a trop oublié », affirme l'économiste et constituant Boislandry, «le grand principe consacré par les écrivains de tous les siècles, et adopté par les Américains, nos maîtres en liberté, que les propriétaires seuls ayant un intérêt immédiat au maintien d'une Constitution, c'est aussi dans la propriété seule que peut résider le pouvoir $\gg^{25}$.

L'abbé Sieyès a été un lecteur assidu des physiocrates, et sa théorie politique s'est construite à la fois avec et contre eux. Il s'est opposé à l'idée que seuls les propriétaires fonciers puissent être considérés comme citoyens actifs et la constitution de 1791 ne retient d'ailleurs pas ce critère, mais celui d'un montant d'impôt acquitté ${ }^{26}$. Les citoyens éligibles devaient cependant être des propriétaires payant une contribution fiscale élevée (article 7 de la section II), disposition que l'on retrouve dans la constitution de l'an III $(1795)^{27}$. Lors des débats qui ont présidé à l'établissement de cette dernière, Sieyès a d'ailleurs tenté d'imposer un «jury constitutionnaire », ancêtre du conseil constitutionnel et organe directement issu de la réflexion de la physiocratie autour du contrôle de conformité des lois avec l'ordre naturel ${ }^{28}$. On comprend mieux, dès lors, que si des débats autour de la progressivité de l'impôt ont eu lieu au cours de la Révolution, «rien de tangible ne fut finalement adopté » sur ce point (p.

\footnotetext{
${ }^{23}$ Archives Parlementaires de 1787 à 1860, Paris, Dupont, t. 8, p. 453. Voir Dan EDELSTEIN, On the Spirtit of Rights, Chicago, The University of Chicago Press, 2019.

${ }^{24}$ Sur l'opposition aux assignats des derniers physiocrates et de leurs émules, le papier-monnaie étant vu comme une attaque frontale contre la propriété privée (les montants des transactions prévus par contrat deviennent caducs du fait de la perte de la valeur des assignats), voir Arnaud ORAIN, La politique du merveilleux. Une autre histoire du Système de Law (1695-1795), Paris, Fayard, 2018, p. 308-321.

${ }^{25}$ Nicolas BERGASSE, Lettre de M. Bergasse, député de la sénéchaussée de Lyon, à ses commettants, Paris, 1790 , p. 23 ; Antoine-François DELANDINE, Faut-il des assignats-monnoie ou des quittances de finances pour la liquidation de la dette publique?, Paris, Imprimerie Nationale, 1790, p. 7 ; Louis DE BOISLANDRY, Considérations sur le discrédit des assignats, Paris, Imprimerie Nationale, 1790, p. 18.

${ }^{26}$ K. BAKER, Inventing the French Revolution, op. cit., p. 245-246.

27 Thierry DEMALS, «Une économie politique de la nation agricole sous la Constituante ?», Revue française d'histoire des idées politiques, 20-2, 2004, p. 83-109.

${ }^{28}$ L. CHARLES et Ph. STEINER, «Entre Montesquieu et Rousseau », art. cit., p. 139-143.
} 
140-141 $)^{29}$. Au contraire, la contribution foncière proportionnelle créée par la Révolution était en rapport direct avec l'impôt unique sur les propriétaires de terre conçu par les physiocrates. Dupont a même vu dans cette taxe, qui devait représenter les trois quarts des impôts directs, un véritable aboutissement du combat qu'il menait depuis vingt-cinq ans ${ }^{30}$.

En tant que groupe et force de propositions économiques, la physiocratie a été violemment combattue, et elle est sortie discréditée, par son fanatisme libéral, des débats des années $1760-1770^{31}$. Les conceptions du droit naturel et la théorie politique du mouvement ont cependant durablement marqué la génération des révolutionnaires. Tocqueville est sans doute celui qui a le mieux résumé cet état de fait. «Les économistes [physiocrates]», dit-il, «ont eu moins d'éclat dans l'histoire que les philosophes ; moins qu'eux ils ont contribué peut-être à l'avènement de la Révolution ; je crois pourtant que c'est surtout dans leurs écrits qu'on peut le mieux étudier son vrai naturel».

Les élites de la fin du XVIII ${ }^{\mathrm{e}}$ siècle étaient largement acculturées aux conceptions de l'économie et de la propriété privée développées par les physiocrates ${ }^{32}$. Mais sous la Révolution, la petite voix discordante de Forbonnais n'était pas tout à fait éteinte. Très critique vis-à-vis de l'impôt foncier proportionnel, il mettait de nouveau en avant ses idées de progressivité fiscale, sur le revenu ou sur les consommations, et expliquait que «quoiqu'il soit théoriquement vrai qu'essentiellement toute imposition se termine, en dernière analyse, en contribution territoriale » comme le pense les physiocrates, «il n'est pas conforme à la raison »d'appliquer une telle contribution. S'appuyant sur l'état de l'économie française dans son ensemble, l'économiste voulait «donner encore un exemple des modifications que l'empire des circonstances apporte nécessairement dans l'application des principes abstraits de l'économie politique ». Trente ans plus tard, l'antiphysiocrate se battait contre les inégalités, et en faveur des petits propriétaires et des pauvres, pour «égaliser le fardeau de la dépense publique », cet objet essentiel de «la justice distributive », grâce à une conception

\footnotetext{
${ }^{29}$ Voir N. Delalande, Les batailles de l'impôt. Consentement et résistances de 1789 à nos jours, Paris, Seuil, 2011, chapitre 1.

${ }^{30}$ Pierre-Henri GoutTE, «Présentation du 'Mémoire lu au Comité des impositions le 26 février 1790' par André Morellet », Revue française d'histoire des idées politiques, 20-2, 2004, p. 149-176.

${ }^{31}$ Arnaud ORAIN, «On the Difficulty of Constituting an Economic avant-garde in the French Enlightenment », The European Journal of the History of Economic Thought, 22-3, 2015, p. 349-358.

32 Alexis DE Tocqueville, L'Ancien Régime et la Révolution, Paris, Gallimard, 1952 [1856], Chap. 3 ; Philippe STEINER, «Les physiocrates et la Révolution française », Revue française d'histoire des idées politiques, 20-2, 2004, p. 1-3 ; J.-P. JESSENNE et N. VIVIER, « Libérer la terre ! », art. cit., p. 46.
} 
historicisée et politique des savoirs économiques ${ }^{33}$. C'est celle que Thomas Piketty défend ${ }^{34}$, et il y a fort à parier qu'elle concurrencera toujours plus fortement à l'avenir l'approche encore dominante de l'économie, jamais débarrassée de ses oripeaux transcendants et naturalistes.

\author{
Arnaud ORAIN \\ LED, Université Paris 8 et IAS Princeton \\ Institut d'Études Européennes - Université Paris 8 \\ 2, rue de la Liberté \\ 93200 Saint-Denis \\ arnaud.orain@univ-paris8.fr
}

\footnotetext{
${ }^{33}$ F. VERON DE FORBONNAIS, «Cahier de doléances et observations que prennent la liberté de présenter au Roi, notre Sire, les Gens du Tiers-État de la paroisse de Champaissant », in Gabriel FLEURY, François Véron de Fortbonnais. Sa famille, sa vie, ses actes, ses æeuvres, Mamers et Le Mans, Fleury et Saint-Denis, 1915, p. 499520 ; Id., "Mémoire sur le genre de questions dont la science de l'économie politique comporte la solution exacte », Mémoires de l'Institut National des Sciences et des Arts, Paris, Baudouin, an IX, III, p. 481-490. Voir également ses articles dans le journal L'Historien, en particulier le $\mathrm{n}^{\circ} 44$ du 14 nivôse et le n ${ }^{\circ} 61$ du $1^{\text {er }}$ pluviôse an IV (tome II, p. 49-51 et p. 297-300).

${ }^{34}$ Thomas PIKETTY, «Vers une économie politique et historique. Réflexions sur le capital au XXI ${ }^{\mathrm{e}}$ siècle », Annales HSS, 70-1, 2015, p. 125-138.
} 